\title{
Lengths of simple periodic geodesics on two-dimensional Riemannian spheres
}

\author{
Alexander Nabutovsky and Regina Rotman
}

October, 26, 2011

\begin{abstract}
Let $M$ be a Riemannian 2-sphere. A classical result of LyusternikShnirelman asserts the existence of three distinct simple non-trivial periodic geodesics on $M$. In this paper we will prove that the lengths of two of them do not exceed $5 d$ and $10 d$ respectively, where $d$ is the diameter of $M$.
\end{abstract}

\section{Main result.}

Theorem 1.1 Let $M$ be a Riemannian manifold of diameter d diffeomorphic to $S^{2}$. There exist two distinct non-trivial simple periodic geodesics on $M$ such that the length of the first of these geodesics does not exceed $5 d$, and the length of the second of these geodesics does not exceed $10 d$.

Recall that the term "simple" means that the geodesics do not have self-intersections. Our proof of the existence of one simple periodic geodesic of length $\leq 5 d$ essentially combines Lyusternik-Shnirelman ideas with the proof by C. Croke, (see $[\mathrm{Cr}]$ ) of the existence of a non-trivial periodic geodesic of length $\leq 9 d$ on $M$ and its later modifications by M. Maeda, (see $[\mathrm{M}]$ ), who improved this upper bound to $5 d$. We need to make several straightforward geometric observations in order to combine these ideas. This will be explained in Sections 2 and 3. Yet more work is required to prove the existence of the second simple periodic geodesic of length $\leq 10 d$, (see Section 4 ). We do not know if the length of the third simple periodic geodesic can be similarly majorized only in terms of $d$, but are able to reduce the problem to its particular case, when every closed curve of length $\leq 2 d$ is contractible 
to a point by a length-decreasing homotopy, (see Section 5). (In all other cases a desired upper bound exists.)

Note that the authors and, independently, S. Sabourau proved that there exists a (possibly self-intersecting) periodic geodesic of length $\leq 4 d$ on $M$. However, it does not seem that the techniques used in $[\mathrm{NR}]$ and in $[\mathrm{S}]$ can be adapted to produce simple periodic geodesics.

Also, note that ellipsoids that are very close to round spheres have only three simple periodic geodesics. Moreover, the length of the fourth (selfintersecting) periodic geodesic becomes arbitrarily large in comparison with $d$, when the metric approaches the round metric. Therefore the length of the fourth periodic geodesic on $M$ cannot be majorized in terms of $d$.

\section{Lyusternik-Shnirelman proof and some geomet- rical observations.}

Our proof uses the basic ideas from the original proof of the existence of three simple periodic geodesics given by L. Lyusternik and L. Shnirelman. (The original proof ([cf. [L]) contained gaps that were corrected in later work by J. Jost, ( $[\mathrm{J}]$ ) and I. Taimanov ( $[\mathrm{T}])$.) The basic idea of this proof is that one can consider three non-trivial homology classes of the pair $\left(\Pi S^{2}, \Pi_{0} S^{2}\right)$ with coefficients in $Z_{2}$, where $\Pi S^{2}$ is the space of non-parametrized free loops on $S^{2}$, and $\Pi_{0} S^{2}$ is its subspace formed by all constant loops. Namely, one considers a 1-dimensional homology class represented by a 1-cycle that consists of all circles on $S^{2}$ obtained as intersections of the standard $S^{2}$ in $R^{3}$ with planes parallel to the $X Z$-plane, a 2-dimensional cycle that consists of all circles obtained by intersecting $S^{2}$ with all planes parallel to the $Z$-axis, and a 3-dimensional cycle that consists of all circles on $S^{2}$.

The main idea of Lyusternik-Shnirelman is to apply a curve-shortening flow to each of these three cycles. The flow is designed so that simple curves that form those cycles remain simple during the curve-shortening process. Then Lyusternik and Shnirelman prove that either those cycles will "hang" at three (simple) periodic geodesics of the diferent lengths, or if either two of them "hang" at the same periodic geodesic, then there will be a whole critical level with infinitely many simple periodic geodesics of the same length.

In this paper we will use only the first two of these cycles. Our first (almost obvious) observation is that there is an alternative representation of the same homology classes: 
Fix a meridian $m$ on the standard $S^{2}$. For every angle $\alpha \in[0, \pi]$ consider a free loop $l_{\alpha}$ formed by two meridians forming the angle $\alpha$ with $m$. Finally, contract each of the loops $l_{0}$ and $l_{\pi}$ formed by pairs of identical meridians travelled in opposite directions over themselves to points. We obtained a map of $([0,1],\{0,1\})$ into $\left(\Pi S^{2}, \Pi_{0} S^{2}\right)$, which is clearly homotopic to the map that we used above to describe the first of the three LyusternikShnirelman classes.

To see this assume that $m$ is the intersection of $S^{2}$ with the $Y Z$-plane. For every convex digon bounded by $l_{\alpha}$ consider the unique circle $C_{\alpha}$ parallel to the $X Z$-plane inscribed into this digon. The homotopy will continuously deform $l_{\alpha}$ into $C_{\alpha}$ inside the considered digon, (see fig. 1).

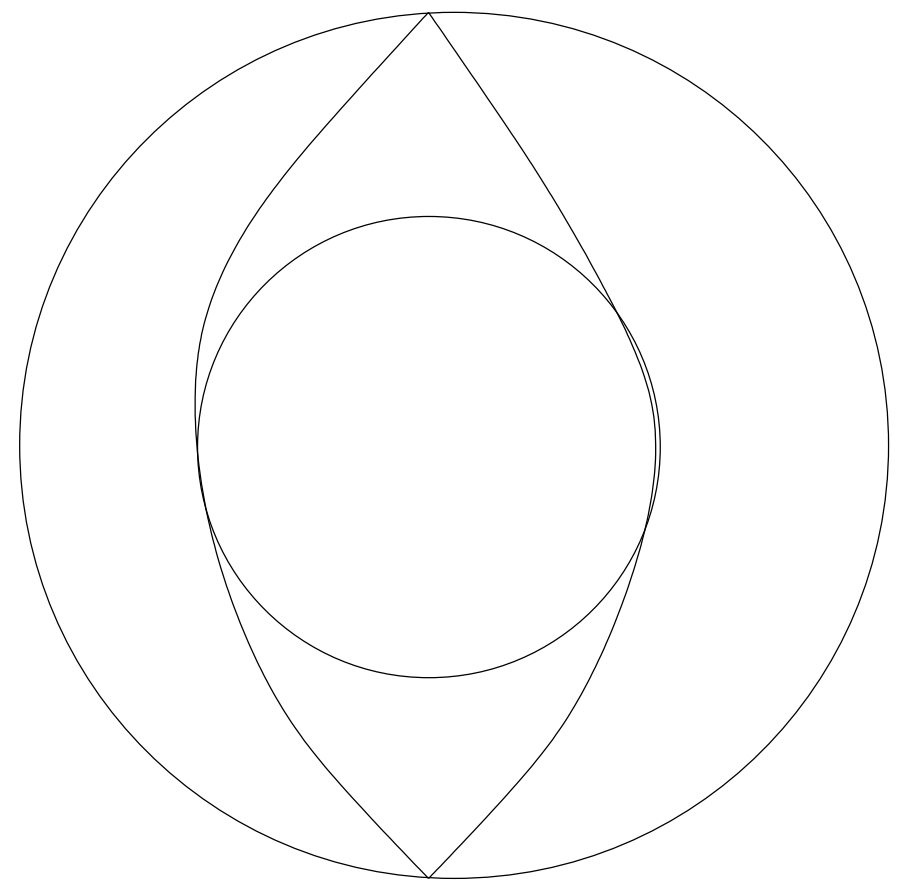

Figure 1:

In order to construct this 1-cycle so that it contains only simple curves we can modify it as follows: Choose any small positive $\varepsilon$. Now the range of $\alpha$ will be $[\varepsilon, \pi-\varepsilon]$. We take curves $l_{\varepsilon}$ and $l_{\pi-\varepsilon}$ and contract them via simple curves using, for example, the Birkhoff curve shortening process on (a round) $S^{2}$. Adding these homotopies to the homotopy between $l_{\varepsilon}$ and $l_{\pi-\varepsilon}$ we obtain the desired relative 1 -cycle $Z_{\varepsilon}^{1}$. 
The 2-dimensional Lyusternik-Shnirelman cycle is obtained by rotating the 1-dimensional Lyusternik-Shnirelman cycle by the angle $\pi$ about $Z$-axis. We can do the same with the 1 -cycle $Z_{\varepsilon}^{1}$ that we constructed above. Thus, we will obtain a relative 2 -cycle $Z_{\varepsilon}^{2}$ representating the Lyusternik-Shnirelman class in $H_{2}\left(\Pi S^{2}, \Pi_{0} S^{2}, Z_{2}\right)$.

There is yet another useful way to represent the 1-dimensional homology class of $Z_{\varepsilon}^{1}$. Fix again a meridian $m$ on $S^{2}$ and an arbitrary (small) $\varepsilon \in(0, \pi)$. Consider the same homotopy starting at a point, ending at $l_{\varepsilon}$, and passing through simple curves that was used in the construction of $Z_{\varepsilon}^{1}$. Then keep one of the meridians forming $l_{\varepsilon}$ fixed and rotate the other one by the angle that vary between 0 and $2 \pi-2 \varepsilon$. At the end of this homotopy we will get a thin digon that can be contracted via simple closed curves to a point, for example, using the Birkhoff curve shortening process. Denote the resulting relative 1-cycle in $\left(\Pi S^{2}, \Pi_{0} S^{2}\right)$ by $Y_{\varepsilon}^{1}$.

Now assume that $f: S^{2} \longrightarrow M$ is a diffeomorphism or, more generally, a piecewise-smooth homeomorphism. It maps simple closed curves on $S^{2}$ into simple closed curves on $M$, and cycles $Z_{\varepsilon}^{1}, Z_{\varepsilon}^{2}, Y_{\varepsilon}^{1}$ into relative cycles in $\left(\Pi M, \Pi_{0} M\right)$. Let $L$ be an upper bound for the images of all meridians of $S^{2}$ under $f$, and $L_{0}$ denote the length of $f(m)$. Then for each positive $\delta$ one can choose a sufficiently small positive $\varepsilon$ to ensure that the lengths of all curves in $f\left(Z_{\varepsilon}^{1}\right), f\left(Z_{\varepsilon}^{2}\right)$ do not exceed $2 L+\delta$ and the lengths of all curves in $f\left(Y_{\varepsilon}^{1}\right)$ does exceed $L+L_{0}+\delta$.

As a corollary, we can conclude that the lengths of two distinct simple periodic geodesics on $M$ do not exceed $2 L$, and, moreover, the length of one of them does not exceed $L+L_{0}$. As a corollary we obtain the first part of the following proposition:

Proposition 2.1 A. Assume that there exists a piecewise-smooth homeomorphism from $S^{2}$ to $M$ that maps all meridians of $S^{2}$ into curves of length $\leq L$ on $M$. Assume that the length of one of these curves does not exceed $L_{0}(\leq L)$. Then there exist two simple periodic geodesics on $M$ of lengths $\leq L_{0}+L$ and $2 L$.

B. Assume that there exists a continuous family of closed curves on $M$ parametrized by $[0,1]$ such that (a) Curves corresponding to 0 and 1 are constant curves; curves corresponding to $t \in(0,1)$ are simple closed curves of length $\leq l$; (b) Different curves do not intersect; and (c) Each point of $M$ is in the image of (exactly) one of these curves. Then there exists a simple periodic geodesic on $M$ of length $\leq l$.

Part B follows from the observation that the continuous family of curves 
provides us with a map $([0,1],\{0,1\}) \longrightarrow\left(\Pi M, \Pi_{0} M\right)$ homotopic to the map considered above and, thus, yielding a simple periodic geodesic of length $\leq l$.

\section{Work of Croke and Maeda and some geometrical observations.}

The following proposition summarizes some facts that were proven in $[\mathrm{Cr}]$ and $[\mathrm{M}]$ :

Proposition 3.1 [Croke-Maeda] Assume that $M$ is a Riemannian manifold diffeomorphic to $S^{2}$ of diameter d. Then either

1. There exists a simple periodic geodesic of length $\leq 2 d$ on $M$;

or

2. There are no simple periodic geodesics of lengths $\leq 2 d$ on $M$, but there exists a non-contractible map $f: S^{1} \longrightarrow \Lambda M$ such that for every $t \in S^{1}$ the length of the closed curve $f(t)$ does not exceed $5 d$. (Here $\Lambda M$ denotes the space of parametrized free loops on $M$. .)

We made the following observation:

Proposition 3.2 If there are no simple periodic geodesics on $M$ of length $\leq$ $2 d$, then for every positive $\varepsilon$ there exists a one-parametric family of pairwise non-intersecting simple closed curves of length $\leq 5 d+\varepsilon$ (and points) swiping out $M$ and satisfying the conditions of Proposition 2.1 B. Also, in this case there exists a map $f: S^{1} \longrightarrow \Lambda M$ such that lengths of images of all meridians of $S^{2}$ do not exceed $5 d+\varepsilon$, the length of the image of at least one meridian is equal to $d$, all meridians are mapped into nonselfintersecting curves, and the images of different meridians intersect only at their endpoints.

This proposition immediately implies the existence of a simple periodic geodesic on $M$ of length $\leq 5 d$. Yet some further work will be required to establish an upper bound for the length of the second simple periodic geodesic.

Proof of Proposition 3.2. We are going to examine the basic ideas of the approach of Croke. First, he uses Berger's lemma to conclude that if $x$ and $y$ are two points such that the distance between them is equal to the diameter 
of the manifold, then there exists a finite collection of minimal geodesics $g_{1}, \ldots, g_{N}$ connecting $x$ and $y$ such that for every $i g_{i}$ and $g_{i+1(\bmod N)}$ form a digon with both angles $<\pi$ unless $x$ and $y$ are both located on a periodic geodesic. (Note that $g_{i}$ do not pairwise intersect.) As the digonal domains are convex, the classical Birkhoff curve shortening process contracts the boundary $\partial D_{i}=g_{i} \bigcup g_{(i+1) \bmod N}$ of each of those domains $D_{i}$ either to a simple non-trivial periodic geodesic of length $\leq 2 d$ or to a point via convex curves contained inside $D_{i}$. (See $[\mathrm{Cr}]$ for a good description of the Birkhoff curve-shortening process and an explanation of the last assertion.)

If the process ends at a simple periodic geodesic, then its length does not exceed $2 d$, which contradicts the assumptions of Proposition 3.2. So, we can assume that $\partial D_{i}$ is contracted to a point. Now our goal is to combine all these homotopies into one family of closed curves.

Consider one of those homotopies that contracts a closed curve $\partial D_{i}$ to a point via curves $h_{t}, t \in[0,1]$, where $h_{0}=\partial D_{i}$, and $h_{1}$ is a constant curve. Denote the value of $h_{1}$ by $z$. The curves $h_{t}$ are convex and have length $\leq 2 d$. Also, the homotopy is monotonous in the following sense: if $t_{1}<t_{2}$, then the domain bounded by $h_{t_{2}}$ containing $h_{1}$ is contained in the domain bounded by $h_{t_{1}}$ that contains $h_{1}$. Let $w$ be a point on $\partial D_{i}$ that is the closest to $z$, (see fig. 2).

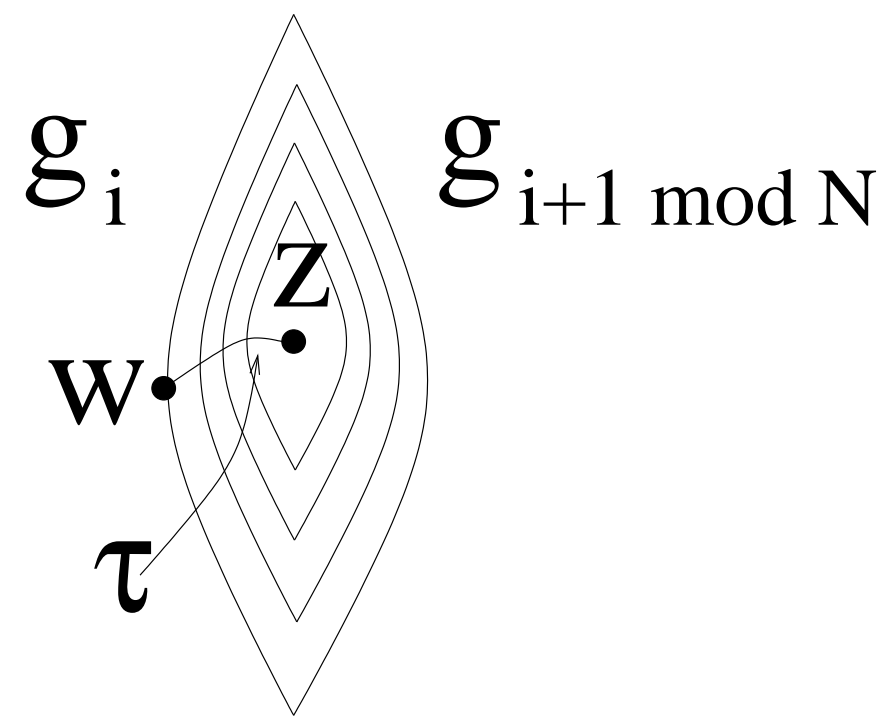

Figure 2: 
The free loop homotopy can be transformed into a path homotopy that contracts $h_{0}=\partial D_{i}$ to $w$ and passes only through loops of length $\leq 2 d+$ $2 \operatorname{dist}(w, z)$ based at $w$ as follows: Connect $w$ and $z$ by a minimal geodesic $\tau$ parametrized by $[0,1]$ interval. Note that this geodesic is contained in $D_{i}$. For every $t$ denote the restriction of $\tau$ to $[0, t]$ by $\tau_{t}$.

It is easy to prove using the convexity of all curves $h_{t}$ and the monotonicity of the homotopy that $\tau(t) \in h_{s}$ if and only if $s \in\left[\alpha_{1}(t), \alpha_{2}(t)\right]$, where for all but countably many values of $t \alpha_{1}(t)=\alpha_{2}(t)$, and $\alpha_{1}\left(t_{1}\right)>\alpha_{2}\left(t_{2}\right)$ if $t_{1}>t_{2}$. (Many details can be found in [M]. See, in particular, proofs of Lemmae 6,7 there). Now we can construct the desired path homotopy by using $\tau_{t} * h_{\alpha_{1}(t)} * \tau_{t}^{-1}$ at the points, where $\alpha_{1}(t)=\alpha_{2}(t)$, and all loops $\tau_{t} * h_{s} * \tau_{t}^{-1}$ for all $s \in\left[\alpha_{1}(t), \alpha_{2}(t)\right]$ at points, where $\alpha_{1}(t)<\alpha_{2}(t)$. To be more precise we are using curves $h_{\alpha(t)}$ reparametrized so that they start and end at $\tau_{t}(t)$. (Of course, eventually we will forget about parametrizations and will end up with a 1-parametric family of non-parametrized curves anyway.) The total time of the homotopy will not exceed 2. It then can be reparametrized by $[0,1]$. Denote these loops by $l_{t}$. The loop $l_{1}$ consists of two copies of $\tau$ travelled in the opposite directions, and we can contract it to the point $w$ through loops $l_{1+t}=\tau_{t} * \tau_{t}^{-1}, t \in[0,1]$. The loops $l_{t}, t \in[0,2]$, form the desired homotopy.

Note that the homotopy $l_{t}$ does not pass through simple closed curves because we go along $\tau_{t}$ twice (in the opposite directions). However, this homotopy can be easily modified to avoid this problem: At each moment of time we could return back not along $\tau_{t}$ but along a curve that goes very closely to $\tau_{t}$ and has almost the same length. At the moment $t=1$ we would end up with a very thin "finger" of thickness much less than the injectivity radius of $M$ that can be contracted through simple loops based at $z$ in an obvious way.

Our next observation is that this homotopy can be used to construct a path homotopy between two geodesic segments $g_{i}$ and $g_{(i+1) \bmod N}$ forming the boundary of $D_{i}$, (see fig. 3).

Assume that $w \in g_{i}$ (the case, when $z \in g_{(i+1) \bmod N}$ is treated similarly). Let $g^{1}$ and $g^{2}$ denote segments of $g_{i}$ between $x$ and $w$, and $w$ and $y$, correspondingly. The path homotopy will include paths $g^{1} * \bar{l}_{1-t / 2} * g^{2}, t \in[0,2]$, , where $\bar{l}_{1-t / 2}$ denotes $l_{1-t / 2}$ travelled in the opposite direction. Note that $g^{1} * \bar{l}_{0} * g^{2}=g^{1} *\left(g^{1}\right)^{-1} * g_{(i+1) \bmod N} *\left(g^{2}\right)^{-1} * g^{2}$, and this curve can be path homotoped along itself to $g_{(i+1) \bmod N}$. Again, the paths in this homotopy are self intersecting as they contain pairs of segments going in opposite 


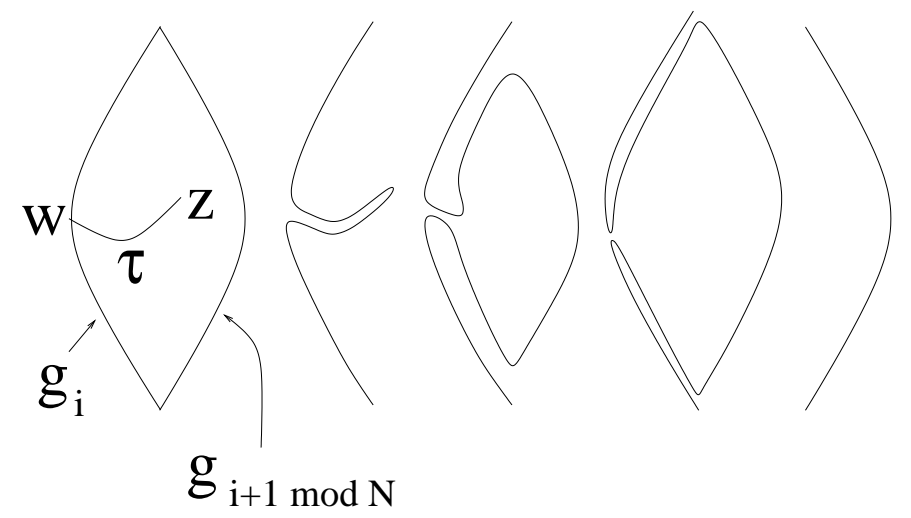

Figure 3: Path homotopy between $g_{i}$ and $g_{i+1} \bmod N$

directions that are then being cancelled. But, as above, it is obvious how to perturb this homotopy to make all curves nonself-intersecting by having pairs of segments that go closely to each other rather than coincide, and contracting long thin fingers using a continous family of minimal geodesics between their corresponding points. The lengths of the curves in this path homotopy do not exceed $3 d+2 d i s t(w, z)+\varepsilon$, where $\varepsilon$ can be made arbitrarily small.

M. Maeda (see $[\mathrm{M}]$ ) noticed that for all digons $D_{i}$ but at most one for every $z \in D_{i}$ there exists $w \in \partial D_{i}$ such that $\operatorname{dist}(w, z) \leq \frac{d}{2}$. (Indeed, otherwise the distance between points $z_{1}, z_{2}$ in the two digons that violate this inequality should have been greater than $d$, which is impossible.) The distance between $z$ and the closest point of the boundary of the exceptional digon (if it exists) does not exceed $d$.

Without any loss of generality we can assume that $D_{1}$ is the exceptional domain, if such a domain exists. According to our assumption its boundary can be contracted to a point by the Birkhoff curve-shortening homotopy inside $D_{1}$. We can perform this homotopy in reverse to obtain $\partial D_{1}$ starting from a point. The lengths of curves during this homotopy do not exceed $2 d$.

Now we are going to extend this homotopy, so as to make it swipe $D_{2}$, and end with a closed curve formed by $\gamma_{1}$ and $\gamma_{3}$. For this purpose we keep $\gamma_{1}$ but vary $\gamma_{2}$ so that it runs over the 1-parametric family of curves $\gamma_{t}$ of length $\leq 4 d+\varepsilon$ in a path homotopy between $\gamma_{2}$ and $\gamma_{3}$ constructed as above. Then we can either sweep-out $D_{3}$ by moving $\gamma_{3}$ using the path homotopy between $\gamma_{3}$ and $\gamma_{4}$, or, if we prefer, move $\gamma_{1}$ to $\gamma_{N}$ (keeping $\gamma_{3}$ fixed). We can continue in this way until all domains $D_{i}$ but one will be 
swept-out. We can arrange for this last domain to be any domain but $D_{1}$. (This observation is not used in the proof but will be important in the next section.) Then we contract the boundary of this domain to a point using the Birkhoff curve-shortening process.

This proves the first assertion of the proposition. To prove the second assertion we proceed as above, but do not use Birkhoff curve-shortening process to contract the boundaries of $D_{1}$ and the last domain. Instead, we construct path homotopies between "halves" of the boundary of $D_{1}$ (and the last domain) exactly as it was done for all other domains. As $\operatorname{dist}(z, w)$ for $D_{1}$ can be as large as $d$ (instead of $\frac{d}{2}$ for other domains) we obtain an extra summand $d$, and the lengths of meridians swiping $D_{1}$ will be bounded by $5 d+\varepsilon$ (but not by $4 d+\varepsilon$ as in other domains).

\section{The length of the second simple periodic geode- sic.}

As we noticed before, this proposition immediately implies the existence of one simple periodic geodesic of length $\leq 5 d$. But its proof implies more than that. First, note that if the Birkhoff curve shortening process contracts the boundary of every domain $D_{i}$ to a point, then, as we saw in Section 1 , we obtain also a second simple periodic geodesic of length $\leq 2(5 d+\varepsilon)$ for an arbitrary small $\varepsilon$, and, thus, a second simple periodic geodesic of length $\leq 10 d$. Second, if the Birkhoff curve shortening process contracts boundaries of two different domains $D_{i}$ to non-trivial periodic geodesics, then these geodesics must be distinct and have length $\leq 2 d$. Finally, assume that the Birkhoff curve-shortening process contracts boundaries of all domains $D_{i}$ but one to points, and it contracts the boundary of the remaining domain that we will denote by $D_{j}$ to a non-trivial periodic geodesic $\gamma$. This geodesic must be a simple geodesic of length $\leq 2 d$ contained in $D_{j}$. It provides a local minimum of the length functional on the space of simple closed curves on $M$. Denote the domain bounded by $\gamma$ inside $D_{j}$ by $D$. The proof of Proposition 3.2 given above implies that there is a slicing of $M \backslash D_{j}$ into simple closed curves (and a point) starting from a point and ending at $\partial D_{j}$. All curves in this 1-parametric family have length $\leq 5 d+\varepsilon$. We can extend this family by applying the Birkhoff curve-shortening process to $\partial D_{j}$ until we get stuck at $\gamma$. Assume that there exists a homotopy $H$ that contracts $\gamma$ to a point within $D$ via simple closed curves of length $\leq L$, where $L$ is some number greater than or equal to $5 d$. Then we can extend our original homotopy by 
$H$, and will obtain a chain representing the 1-dimensional homology cycle in the space of non-parametrized simple closed curves considered in section 1 such that all closed curves have length $\leq L$. Therefore there exists a simple closed geodesic of length $\leq L$ in $M$ that corresponds to this cycle, and so is not a local minimum of the length functional on the considered space. Thus, this geodesic is different from $\gamma$. Hence, Theorem 1 would follow from the following lemma:

Lemma 4.1 Let $D$ be a domain on $M$ bounded by a simple periodic geodesic $\gamma$ of length $\leq 2 d$. Assume that there are no simple periodic geodesics of length $\leq 4 d$ inside $D$ providing a local minimum for the length functional. Then for every positive $\varepsilon$ there exists a homotopy contracting $\gamma$ to a point and passing via simple closed curves of length $\leq 8 d+\varepsilon$.

Proof. The crucial observation is the following relative version of Berger's lemma: Let $z$ be a point in $D$ maximizing the distance to $\gamma=\partial D$. Then for every non-zero vector $v$ in the tangent space to $M$ at $z$ there exists a shortest path $\tau$ from $z$ to $\partial D$ such that its tangent vector at $z$ makes angle $\leq \pi / 2$ with $v$. This assertion can be proven by contradiction exactly as Berger's lemma: Assume that the assertion is not true for some vector $v$. Move $z$ in the direction of $v$ for a very small distance $\delta$. Connect the resulting point $z_{\delta}$ with $\partial D$ by a geodesic of minimal length. Passing to the limit as $\delta \longrightarrow 0$ and choosing an appropriate subsequence we will find a sequence of geodesics of length $<\operatorname{dist}(z, \partial D)$ starting at $z_{\delta}$, ending at $\partial D$ and converging to a shortest geodesic $\varrho$ between $z$ and $\partial D$. The first variation formula for lengths of geodesics and the fact that the angle between $\varrho$ and the minimal geodesic $z z_{\delta}$ is obtuse lead to a contradiction proving the assertion.

Thus, one can choose a finite set of shortest paths leading from $z$ to $\partial D$ such that the angle between each consecutive pair of these paths is $\leq \pi$. These paths do not intersect other than at the endpoints, have length $\leq d$ and divide $D$ into a finite collection of convex domains $T_{i}$. The Birkhoff curve-shortening process applied to the boundary of each domain $T_{i}$ contracts it to a point as we assumed that there are no simple closed geodesics of length $\leq 4 d$ ( $\geq$ length $\partial T_{i}$ ), (see fig. 4).

Now we can construct the desired homotopy by contracting $\gamma=\partial D$ combining homotopies contracting $\partial T_{i}$ almost exactly as it was done in the proof of Proposition 3.2. We omit the details of the process. 


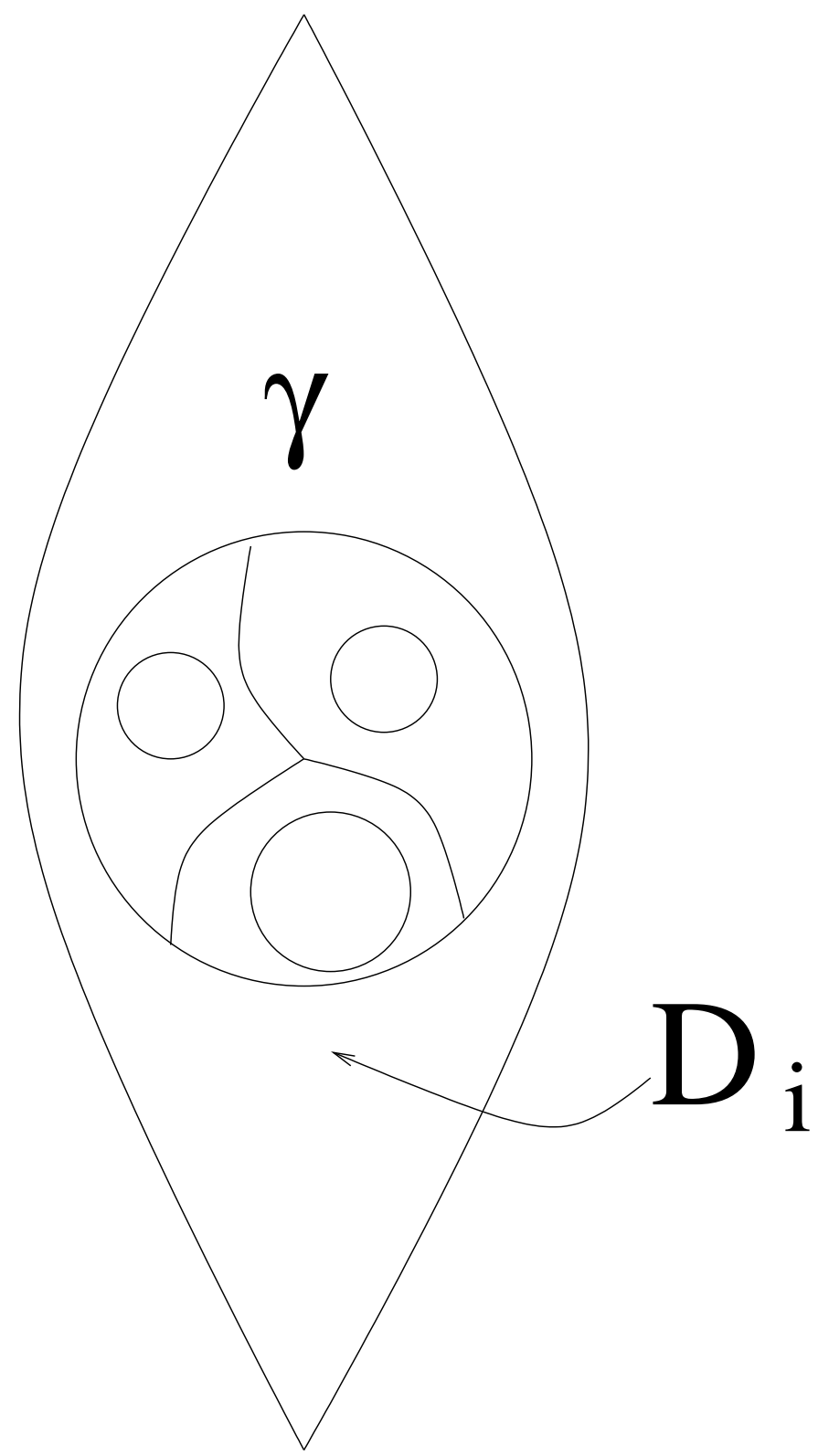

Figure 4: Contracting closed geodesic $\gamma$ 


\section{The length of the third simple periodic geodesic.}

Can we prove an upper bound of the form const $d$ for the length of the third simple periodic geodesic in Lyusternik-Shnirelman theorem?

In the previous section we saw that there are three possibilities: (i) There exist two simple periodic geodesics of lengths $\leq 2 d$ and $\leq 4 d$ providing local minima for the length functional on the space of closed curves on $M$; (ii) There exists one simple periodic geodesic of length $\leq 2 d$ providing a local minimum for the length functional on the space of closed curves on $M$, and there exists a non-contractible map of a circle into the space of simple closed curves on $M$ that was constructed in the previous section, such that the lengths of closed curves that are the images of points of this circle do not exceed $\leq 8 d+\varepsilon$. Finally, in the last case (iii) there exist no periodic geodesics of length $\leq 2 d$ locally minimizing the length functional.

In the case (i) we can iterate the argument from section 3 and to conclude that either there exist three simple periodic geodesics of lengths $\leq 8 d$ providing local minima for the length functional, or there exists a slicing of $M$ into meridianal curves of length $\leq$ const $d$ that implies the existence of the desired bound for the length of the third simple periodic geodesic. In the case (ii) we can convert the homotopies contracting boundaries of $D_{1}$ and another domain as free loops into path homotopies between "halves" of the boundaries of these domains as it was done for the other domains during the construction of this homotopy, and, as the result, will obtain two simple periodic geodesics with positive indices of length $\leq$ const $d$.

Thus, the problem stated at the beginning of this section reduces to its particular case, when no closed curve of length $\leq 2 d$ is a local minimum of the length functional, or, equivalently, every closed curve of length $\leq 2 d$ on $M$ can be contracted to a point by a length-decreasing homotopy. Yet we do not know how to solve the problem in this particular case.

Acknowledgements: Both authors acknowledge a partial support of this work by their corresponding NSERC Discovery Grants. We would like to thank an anonymous referee for his comments that helped to improve the exposition.

\section{References}

[Cr] C. B. Croke, Area and the length of the shortest periodic geodesic, J. Differential Geometry, 27(1988), 1-21. 
$[\mathrm{J}] \quad$ J. Jost, A nonparametric proof of the theorem of Lyusternik and Schnirelman, Arch. Math. (Basel), 53(1989), 497-509.

[L] L.A. LyUsternik, Topology of functional spaces and the calculus of variations at large, Trudy Mat. Inst. Steklov 19(1947).

[M] M. MAEDA, The length of a closed geodesic on a compact surface, Kyushu J. Math. 48(1994), 9-18.

[NR] A. Nabutovsky, R. Rotman, The length of the shortest closed geodesic on a 2-dimensional sphere, Int. Math. Res. Not., 2002, No. 23, 1211-1222.

[T] I. A. Taimanov, Closed extremals on two-dimensional manifolds, Russian Math. Surveys 47(1992), 163-211.

[S] S. Sabourau, Filling radius and short closed geodesics of the 2-sphere, Bull. Math. Soc. France, 132(2004), 105-136.

Alexander Nabutovsky

Department of Mathematics

University of Toronto

Toronto, Ontario M5S 2E4

Canada

e-mail: alex@math.toronto.edu
Regina Rotman

Department of Mathematics

University of Toronto

Toronto, Ontario M5S 2E4

Canada

rina@math.toronto.edu 